\title{
Prioritizing pediatric chronic pain and comprehensive pain treatment in the context of the opioid epidemic
}

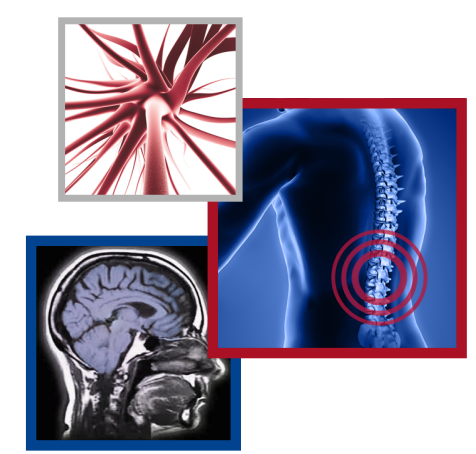

\author{
Sarah R Martin*,1 \& Lonnie K Zeltzer ${ }^{1}$ \\ ${ }^{1}$ Pediatric Pain \& Palliative Care Program, Division of Pediatric Hematology-Oncology, Department of Pediatrics, David Geffen \\ School of Medicine at UCLA \\ * Author for correspondence: srmartin@mednet.ucla.edu
}

First draft submitted: 23 December 2017; Accepted for publication: 09 January 2018; Published online: 16 February 2018

Keywords: children $\bullet$ chronic pain $\bullet$ complementary therapy $\bullet$ opioids $\bullet$ pain $\bullet$ pain management $\bullet$ pediatric pain - pharmacology • policy issues • psychology

The USA is faced with a surge in opioid abuse and misuse and the President's Commission on Combating Drug Addiction and the Opioid Crisis recently released a report that outlines their perspective on the current problem and specific recommendations. As pain and opioid policies are developed, pain clinicians and researchers are uniquely poised to educate policy makers on the current state of pediatric pain management research and practice to ensure the development of effective policies for children and adolescents. The current editorial, adapted from a Pain Action Alliance to Implement a National Strategy (PAINS) Policy and Educational Brief [1], summarizes the state of pediatric chronic pain management and suggests recommendations for policy development. Pediatric chronic pain management is highlighted, as it is often absent from policy and funding initiatives despite its prevalence, economic impact and relevance to the current opioid epidemic. Further, policy proposals thus far have focused on substance abuse and prescription monitoring and, although addressing these issues is important, we propose that a failure to recognize the current lack of appropriate pediatric pain management education and treatment will only further perpetuate the need for opioid abuse treatment.

\section{Pediatric chronic pain}

Chronic pain is a complex, biopsychosocial condition and studies estimate that 5-38\% of youth suffer from chronic pain [2-4] and, from 1994 to 2007, pediatric opioid prescription rates doubled. Pediatric chronic pain is associated with a significant level of economic burden in this country. Recent studies estimate that the economic cost (i.e., healthcare costs, lost employment and out-of-pocket expenses) of pediatric chronic pain is US\$19.5 billion annually [5] and it accounts for US $\$ 11.8$ billion in total healthcare expenditures specifically (i.e., healthcare expenditures paid by insurance companies and families), which is more than expenditures associated with obesity (US $\$ 0.73$ billion) and asthma (US $\$ 9.23$ billion) combined [6].

Poor pain management in childhood not only affects the quality of life of children and their families, but also puts children at-risk for continued and heightened impairment as they move into adulthood. Unfortunately, up to $73 \%$ of children and adolescents with chronic pain will continue to have pain in adulthood and are likely to develop new pain conditions $[7,8]$.

\section{Evidence-based shift in pediatric chronic pain management}

In 2011, the Institute of Medicine called for a 'cultural transformation' in the way pain is perceived, judged and treated' [9]. The healthcare industry has a strong attachment to the biomedical approach to treating disease, which prioritizes identifying and treating an injury, tissue damage or other observable physical pathology. Decades of pain research have taught us that the experience of pain is complex, not simply directly related to the injury or damage sustained, and chronic pain can often occur in the absence of identified physical pathology, a factor that presents problems for providers working within a biomedical framework. 
The experience of pain, regardless of the cause, is modulated by the same areas of the brain that process not only sensory input, but also attention, emotion, fear, memory and behavior, to name a few [10]. Thus, biological, psychological and social factors, including the family context and caregiver behaviors [11], all have the potential to alter a child's pain experience.

Fortunately, after decades of pediatric pain research, we know that: humans of all ages experience pain; poorly managed pediatric pain increases the risk for chronic pain continuing into adulthood; and effective pain treatment includes a combination of biomedical, psychosocial, physical rehabilitation and complementary modalities. Although the first point is now widely accepted, education, funding and policy to address the latter points are lacking. Given that chronic pain is maintained through complex biopsychosocial mechanisms, it needs to be treated with comprehensive pain management that should be tailored to each child's individual needs and family context and include both pharmacological and non-pharmacological approaches. Examples of effective, evidence-based pain management treatments for pediatric chronic pain include: cognitive-behavioral and neuromodulatory modalities (e.g., behavioral exposure/activation, cognitive reframing, hypnosis, biofeedback and mindfulness), rehabilitation (e.g., occupational and physical therapy), complementary modalities (e.g., acupuncture, chiropractic/massage and yoga), healthy lifestyle behavior (e.g., physical activity, nutrition and sleep hygiene) and medication (e.g., anticonvulsants, anti-inflammatory drugs, opioids, selective serotonin reuptake inhibitors [SSRIs], and serotonin and norepinephrine reuptake inhibitors [SNRIs]) [12-15].

Comprehensive pain management treatments are not only effective in treating pain and reducing the risk of continued pain into adulthood [16], but they also save money. Recent findings show that, when compared with usual care, comprehensive treatment of pediatric chronic pain can result in a US $\$ 27,199$ reduction in annual healthcare costs per family, accounting for the cost of care [17]. In fact, broader research indicates that integrating behavioral health providers into pediatric clinics allows physicians to see $42 \%$ more patients and collect US $\$ 1142$ more revenue per day $[18,19]$.

Given the current state of opioid abuse in the USA, a discussion surrounding the use of opioids in pediatric pain management is warranted. Indeed, opioids can be effective in relieving acute pain and pain flares in certain conditions (e.g., sickle cell disease, cancer and end-stage disease), but they do not correct and may contribute to nervous system dysfunction often present in chronic pain. Given that appropriate management of acute pain can prevent the transition to chronic pain, the use of opioids is often indicated in such cases. However, opioids are only effective when used appropriately and when provided in the context of continued biopsychosocial assessment and care.

\section{Barriers to proper pediatric pain management}

Although many factors prevent children from receiving proper pain treatment and perpetuate the misuse of opioids, we propose that two specific barriers are at the root of the problem. First, there is limited pediatric chronic pain management education in the medical field. Second, there is minimal funding, healthcare insurance coverage and policy directed toward providing comprehensive pediatric pain treatment.

Limited education surrounding the nature and development of chronic pain in children is evident in the healthcare system. Currently, healthcare providers do not receive adequate training in pain management [9]. In a survey of 104 US medical schools, only four schools had a pain course requirement and only one included pediatric pain in the curriculum [20]. The cumulative number of pain teaching hours across schools ranged from 1 to $31 \mathrm{~h}$.

Another key barrier to effective pediatric chronic pain management is a lack of appropriate insurance coverage and policy directed toward providing access to treatment. When children have pain, their caregivers take them to the doctor. Unfortunately, given the statistics stated above, it is likely that the primary care physician will not know how to properly assess and treat the child's pain, especially if no 'identified cause' is discerned. Even if a child is lucky enough to see a physician who has received pediatric chronic pain education, it is likely that access to comprehensive treatment will be limited or nonexistent. Current healthcare policy does not mandate access to comprehensive, non-pharmacological treatments in clinics where chronic pain may be a presenting concern (e.g., primary care, orthopedics, rheumatology, gastroenterology, neurology and oncology/hematology), a significant factor that limits access to appropriate care. If children are lucky enough to live close to one of the few pediatric pain centers in the country, they are often subject to long waitlists because of the high demand for treatment. As children wait for appropriate treatment, their condition will worsen, economic strain on the healthcare system will continue, and they are likely to turn to ineffective and potentially harmful treatments (e.g., unmonitored opioid use). 


\section{Concluding comments}

We commend the members of the President's Commission on Combating Drug Addiction and the Opioid Crisis for including recommendations that address barriers to Medicare and Medicaid Services reimbursement for nonpharmacological pain treatment and encouraging the incorporation of behavioral and psychosocial treatments, as well as advocating for increased NIH funding for prevention research. Although these recommendations could result in a significant improvement in the current approach to care, most of the recommendations, as currently stated, apply to the treatment of substance use disorders, which perpetuates the prioritization of treatment of a disorder that is already present as opposed to a preventive approach that addresses the need for effective pain management. Unfortunately, no recommendations specifically address pediatric pain management. With this in mind, we urge pediatric pain providers and researchers to consider ways in which their work can help encourage the effective treatment of pain in children. Policy makers must be made aware of the importance of implementing policies and funding to ensure access to evidence-based pain management that may prevent youth from suffering from chronic pain and ever needing opioid addiction treatment.

Collectively, we propose that future policies should prioritize evidence-based, biopsychosocial management of pediatric chronic pain as well as enact pediatric pain education initiatives for healthcare providers. In addition, targeted government funding for research on comprehensive, pediatric pain treatment, including cost-effective models of care, is needed. Mandating insurance coverage for evidence-based pediatric pain treatment (i.e., coordinated physical, psychological and complementary treatment) and requiring pediatric treatment centers to comply with pediatric pain treatment recommendations would help ensure access to preventative, evidence-based pain care. Finally, pediatric pain clinicians and researchers have produced valuable evidence that is often not consulted during policy development. Thus, more efforts are needed to support evidence-based pain management advocacy and knowledge translation.

\section{Financial \& competing interests disclosure}

The authors have no relevant affiliations or financial involvement with any organization or entity with a financial interest in or financial conflict with the subject matter or materials discussed in the manuscript. This includes employment, consultancies, honoraria, stock ownership or options, expert testimony, grants or patents received or pending, or royalties.

No writing assistance was utilized in the production of this manuscript.

\section{References}

1. Martin SR, Zeltzer LK. Pediatric chronic pain: prevalence, economic impact, and its relevance to the current opioid epidemic. The Pain Action Alliance to Implement a National Strategy (PAINS). http://painsproject.org/pediatric-chronic-pain-brief/

2. Huguet A, Miró J. The severity of chronic pediatric pain: an epidemiological study. J. Pain 9(3), 226-236 (2008).

3. King S, Chambers CT, Huguet A et al. The epidemiology of chronic pain in children and adolescents revisited: a systematic review. Pain 152(12), 2729-2738 (2011).

4. Perquin CW, Hazebroek-Kampschreur AAJ, Hunfeld JA et al. Pain in children and adolescents: a common experience. Pain 87(1), 51-58 (2000).

5. Groenewald CB, Essner BS, Wright D, Fesinmeyer MD, Palermo TM. The economic costs of chronic pain among a cohort of treatment-seeking adolescents in the United States. J. Pain 15(9), 925-933 (2014).

6. Groenewald CB, Wright DR, Palermo TM. Health care expenditures associated with pediatric pain-related conditions in the United States. Pain 156(5), 951-957 (2015).

7. Walker LS, Dengler-Crish CM, Rippel S, Bruehl S. Functional abdominal pain in childhood and adolescence increases risk for chronic pain in adulthood. Pain 150(3), 568-572 (2010).

8. Brna P, Dooley J, Gordon K, Dewan T. The prognosis of childhood headache: a 20 year follow-up. Arch. Pediatr. Adolesc. Med. 159(12), 1157-1160 (2005).

9. Institute of Medicine (US) Committee on Advancing Pain Research, Care, and Education. Relieving Pain in America: a Blueprint for Transforming Prevention, Care, Education, and Research. National Academies Press, DC, USA (2011).

10. Simons L, Elman I, Borsook D. Psychological processing in chronic pain: a neural systems approach. Neurosci. Biobehav. Rev. 39, 61-78 (2014).

11. Palermo TM, Holley AL. The importance of the family environment in pediatric chronic pain. JAMA Pediatr. 167(1), 93-94 (2013).

12. Fisher E, Heathcote L, Palermo TM, de C Williams AC, Lau J, Eccleston C. Systematic review and meta-analysis of psychological therapies for children with chronic pain. J. Pediatr. Psychol. 39(8), 763-782 (2014). 
13. Hechler T, Kanstrup M, Holley AL et al. Systematic review on intensive interdisciplinary pain treatment of children with chronic pain. Pediatrics 136(1), 115-127 (2015).

14. Simons LE, Basch MC. State of the art in biobehavioral approaches to the management of chronic pain in childhood. Pain Manag. 6(1), 49-61 (2016).

15. Groenewald CB, Beals-Erickson SE, Ralston-Wilson J, Rabbitts JA, Palermo TM. Complementary and alternative medicine use by children with pain in the United States. Acad. Pediatr. 17(7), 785-793 (2017).

16. Zernikow B, Ruhe A-K, Stahlschmidt L et al. Clinical and economic long-term treatment outcome of children and adolescents with disabling chronic pain. Pain Med. 19(1), 16-28 (2017).

17. Evans JR, Benore E, Banez GA. The cost-effectiveness of intensive interdisciplinary pediatric chronic pain rehabilitation. J. Pediatr. Psychol. 41(8), 849-856 (2015).

18. Gouge N, Polaha J, Rogers R, Harden A. Integrating behavioral health into pediatric primary care: implications for provider time and cost. South. Med. J. 109(12), 774-778 (2016).

19. Asarnow JR, Rozenman M, Wiblin J, Zeltzer L. Integrated medical-behavioral care compared with usual primary care for child and adolescent behavioral health: a meta-analysis. JAMA Pediatr. 169(10), 929-937 (2015).

20. Mezei L, Murinson BB. Pain education in North American medical schools. J. Pain 12(12), 1199-1208 (2011). 